\title{
Application of bispecific antibody against antigen and hapten for immunodetection and immunopurification
}

\author{
Hyori Kim ${ }^{1,2}$, Sunyoung Park ${ }^{1,2}$, Hwa Kyoung Lee ${ }^{1,2}$ and Junho Chung ${ }^{1,2}$ \\ We present a bispecific antibody that recognizes an antigen and a hapten and can be applied to various biological assays, \\ including immunoblotting and immunoprecipitation. In immunoblot analysis of serum, an anti-C5 $\times$ anti-cotinine bispecific \\ tandem single-chain variable fragment (scFv)-Fc fusion protein and cotinine-conjugated horseradish peroxidase (HRP) generated \\ a clean signal without the high background that was observed in a parallel experiment using HRP-conjugated goat anti-rabbit \\ immunoglobulin G (Fc-specific) antibody. In immunoprecipitation analysis of serum, use of the bispecific tandem scFv-Fc fusion \\ protein and cotinine-crosslinked magnetic beads significantly reduced the amount of protein contaminants compared with a \\ parallel experiment done with protein A agarose beads. In subsequent immunoblot analysis, use of cotinine-HRP as the \\ secondary probe instead of HRP-conjugated goat anti-rabbit IgG (Fc-specific) antibody successfully eliminated the band \\ corresponding to the bispecific tandem scFv-Fc fusion protein.
}

Experimental \& Molecular Medicine (2013) 45, e43; doi:10.1038/emm.2013.83; published online 27 September 2013

Keywords: bispecific antibody; hapten; immunoblot; immunoprecipitation

\section{INTRODUCTION}

Antibodies are used in a wide variety of biological experiments such as immunoblot analysis, immunoprecipitation, flow cytometry and affinity purification. In immunoblot analysis and flow cytometry, a secondary antibody labeled with enzyme or fluorescent dye is frequently used to detect or quantify the primary antibody. In immunoprecipitation and affinity purification experiments, protein A is widely employed to immobilize antibodies on a solid support. Reactivity of secondary antibody and protein A with immunoglobulins (Igs) from a range of diverse species frequently raises the issues of background in immunoblot and flow cytometry analysis and of protein contaminants in immunoprecipitation and affinity purification, especially when the sample contains Ig.

To reduce nonspecific binding of secondary antibody or protein A, direct chemical crosslinking of primary antibody to enzyme, fluorescent dyes and solid supports has been used. However, the functional groups on antibody molecules that are involved in crosslinking are randomly selected during the chemical crosslinking procedure. When residues critical for antigen-antibody binding are involved, the antibody loses its reactivity; therefore, it is essential to establish optimal conditions for every crosslinking process, under which a sufficient amount of antibody retains its reactivity while efficient crosslinking takes place. To avoid having to optimize conditions for every crosslinking procedure, biotin-labeled antibody is frequently employed and detected using avidin-conjugated enzymes and fluorescent dyes. For affinity purification of proteins and cells, either biotinlabeled anti-protein antibody or anti-cell surface molecule antibody paired with avidin-crosslinked solid support can be employed. ${ }^{1,2}$ However, even in this system, there remains the issues of background and contamination because of the presence of proteins reactive to biotin and avidin in biological samples. ${ }^{3}$

Molecular biology techniques, including phage display, have recently been broadly adopted for the generation of antibodies. This technology allowed us to obtain the antibody as well as its coding sequence. With this information, we can produce the antibody molecule in various formats, such as single-chain variable fragment $(\mathrm{scFv})$ and $\mathrm{scFv}-\mathrm{Fc}$ fusion protein. Taking advantage of these options, we hypothesized that we might use a hapten and an anti-hapten antibody as a universal linker between an antibody and hapten-conjugated enzymes, fluorescent dyes and solid supports. In this study, to test this hypothesis, we prepared a bispecific antibody reactive to

\footnotetext{
${ }^{1}$ Department of Biochemistry and Molecular Biology, Seoul National University College of Medicine, Seoul, Republic of Korea and ${ }^{2}$ Cancer Research Institute, Seoul National University, Seoul, Republic of Korea

Correspondence: Dr J Chung, Department of Biochemistry and Molecular Biology, Seoul National University College of Medicine, 103 Daehak-ro, Jongno-gu, Seoul 110-799, Republic of Korea.

E-mail: jjhchung@snu.ac.kr
}

Received 23 June 2013; revised 2 July 2013; accepted 4 July 2013 
a

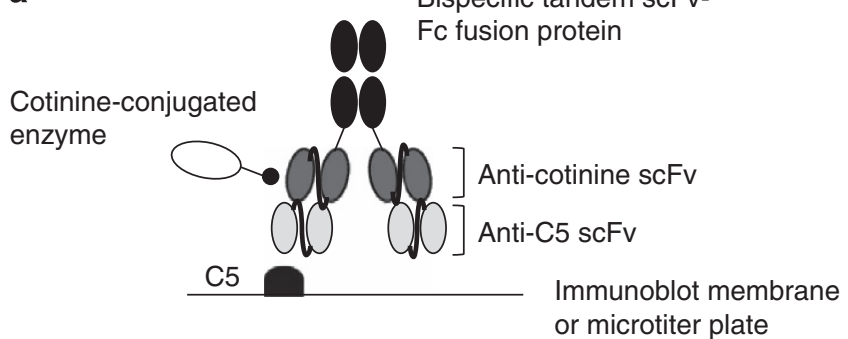

b

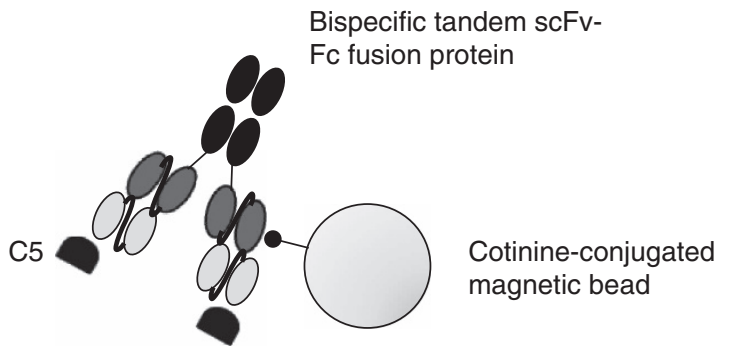

Figure 1 Application of bispecific tandem single-chain variable fragment ( $(\mathrm{CFv})-\mathrm{Fc}$ fusion protein for (a) enzyme immunoassay and immunoblot analysis and (b) immunoprecipitation and immunoaffinity purification.

complement C5 and cotinine (Figure 1). The bispecific antibody was coupled with horseradish peroxidase (HRP)-cotinine and cotinine-conjugated magnetic beads and applied to immunoblot analysis and immunoprecipitation, respectively.

\section{MATERIALS AND METHODS}

Construction of bispecific tandem scFv-Fc fusion vector

A gene encoding the leader sequence of the human Ig $\kappa$-chain, anticomplement C5 scFv (H Kim, HK Lee, WJ Yang, J Chung, unpublished data), a linker consisting of (Gly-Gly-Gly-Ser) ${ }_{3}$, anticotinine $\mathrm{scFv}^{4}{ }^{4}$ the hinge region of human $\mathrm{IgG}_{1}$ and the $\mathrm{C}_{\mathrm{H} 2}-\mathrm{C}_{\mathrm{H} 3}$ domains of rabbit IgG was chemically synthesized (Genscript, Piscataway, NJ, USA) with $K p n I$ and BamHI restriction sites at the $5^{\prime}$ and $3^{\prime}$ ends, respectively. The gene was digested with $K p n I$ and BamHI (both from New England BioLabs, Ipswich, MA, USA) and subjected to $1 \%$ agarose (Invitrogen, Carlsbad, CA, USA) gel electrophoresis, and purified using QIAquick Gel Extraction Kit (Qiagen, Germantown, MD, USA) according to the manufacturer's instructions. The pCEP4 vector (Invitrogen) was also digested with $K p n \mathrm{I}$ and BamHI (both from New England BioLabs) and ligated with the gene encoding the tandem scFv-Fc using T4 ligase (Invitrogen). Ligated products were transformed into calcium chloride competent Escherichia coli DH5 $\alpha$ cells. Plasmid DNA was purified using PureLink HiPure Plasmid Maxiprep Kit (Invitrogen) according to the manufacturer's instructions and stored at $-20^{\circ} \mathrm{C}$ until use.

\section{Cell culture and expression of bispecific tandem scFv-Fc protein}

HEK293F cells (FreeStyle 293-F cells, Invitrogen) were cultured in FreeStyle 293 Expression Medium (Invitrogen) containing 100 units $\mathrm{ml}^{-1}$ penicillin and $100 \mu \mathrm{g} \mathrm{ml}^{-1}$ streptomycin at $37^{\circ} \mathrm{C}$ in an atmosphere containing $7 \% \mathrm{CO}_{2}$ on an orbital shaking incubator (Minitron, INFORS HT, Bottmingen, Switzerland) at 135 r.p.m. The expression vector was transfected into $293-\mathrm{F}$ cells using $25-\mathrm{kDa}$ linear polyethylenimine (Polysciences, Warrington, PA, USA) as reported previously. ${ }^{5}$ Briefly, the mixture of $2 \mu \mathrm{g}$ plasmid DNA and $4 \mu \mathrm{g}$ linear polyethylenimine in $100 \mu 150 \mathrm{~mm} \mathrm{NaCl}$ solution was prepared per $\mathrm{ml}$ of cell culture medium. After a 15-min incubation at room temperature, the mixture was added to HEK293F cells $\left(2 \times 10^{6}\right.$ cells $\mathrm{ml}^{-1}$; Invitrogen $)$ and the cells were grown in FreeStyle 293 Expression Medium for 5 days at $37^{\circ} \mathrm{C}$ in an atmosphere containing $7 \% \mathrm{CO}_{2}$ on an orbital shaking incubator (Minitron) at 135 r.p.m. The fusion protein was purified from culture supernatant by affinity chromatography using protein A agarose beads (RepliGen, Waltham, MA, USA) according to the manufacturer's instructions. After purification, the flow-through and purified fractions were mixed with sample loading buffer (NuPAGE LDS Sample Buffer, Invitrogen) and reducing agent (NuPAGE Sample Reducing Agent, Invitrogen), boiled for $5 \mathrm{~min}$ and electrophoresed through sodium dodecyl sulfate-polyacrylamide gel electrophoresis (SDS-PAGE; NuPAGE 4-12\% Bis-Tris, Invitrogen) gel according to the manufacturer's instructions. The gel was stained with Coomassie Brilliant Blue R-250 (Amresco, Solon, OH, USA).

\section{Conjugation of cotinine to HRP}

Cotinine was conjugated to HRP as described previously. ${ }^{4}$ A mixture containing $17.6 \mathrm{mg} \quad(0.10 \mathrm{mmol})$ trans- $4^{\prime}$-cotininecarboxylic acid (Sigma-Aldrich, St Louis, MO, USA), $13.9 \mathrm{mg} \quad(0.12 \mathrm{mmol})$ $N$-hydroxysuccinimide (Sigma-Aldrich) and $24.3 \mathrm{mg} \quad(0.12 \mathrm{mmol})$ dicyclohexylcarbodiimide (Sigma-Aldrich) in $1 \mathrm{ml}$ dimethylformamide (Sigma-Aldrich) was stirred at room temperature for $2 \mathrm{~h}$. After centrifugation at $10000 \times g$ for $30 \mathrm{~min}$, a $400-\mu \mathrm{l}$ aliquot of clear supernatant containing the active ester was diluted with $500 \mu \mathrm{l}$ dimethylformamide and slowly added to $2 \mathrm{ml} 50 \mathrm{~mm}$ carbonate buffer (pH 9.6) containing $10 \mathrm{mg} \mathrm{ml}^{-1} \mathrm{HRP}$. This mixture was allowed to react at room temperature for $3 \mathrm{~h}$ with constant stirring. The conjugate was dialyzed against phosphate-buffered saline (PBS) for $12 \mathrm{~h}$ at $4{ }^{\circ} \mathrm{C}$ and stored at $-20{ }^{\circ} \mathrm{C}$ until use.

\section{Enzyme immunoassay of bispecific tandem scFv-Fc fusion protein}

The wells of microtiter plates (Corning Costar, Cambridge, MA, USA) were coated by the addition of $100 \mathrm{ng}$ human complement C5 (Merck Millipore, Darmstadt, Germany) in $20 \mu \mathrm{l} 0.1 \mathrm{~m}$ sodium bicarbonate buffer ( $\mathrm{pH}$ 8.6) and incubated at $4{ }^{\circ} \mathrm{C}$ overnight. Wells were washed with PBS, blocked with PBS containing $1 \%$ skim milk (BD Biosciences, San Jose, CA, USA) at $37^{\circ} \mathrm{C}$ for $1 \mathrm{~h}$ and washed again with PBS. Anti-C5 $\times$ anti-cotinine bispecific tandem $\mathrm{scFv}-\mathrm{Fc}$ fusion protein $\left(1 \mu \mathrm{g} \mathrm{ml}^{-1}\right.$ in PBS containing $1 \%$ skim milk) was diluted twofold and added to each well. An equal volume of PBS containing $1 \%$ skim milk was added to control wells. Plates were incubated at $37^{\circ} \mathrm{C}$ for $2 \mathrm{~h}$ and then washed five times with $0.05 \%$ Tween- 20 (Sigma-Aldrich) in PBS. Wells were incubated with either $50 \mu \mathrm{l}$ cotinine-HRP $\left(1 \mu \mathrm{g} \mathrm{ml}^{-1}\right)$ or HRP-conjugated goat anti-rabbit IgG (Fc-specific) antibody (10 $\mathrm{ng} \mathrm{ml}^{-1}$; Abcam) diluted in $1 \%$ skim milk in PBS at $37^{\circ} \mathrm{C}$ for $1 \mathrm{~h}$ and then washed five times with $0.05 \%$ Tween20 in PBS. Peroxidase activity was detected by the addition of $50 \mu \mathrm{l}$ $3,3^{\prime}, 5,5^{\prime}$-tetramethylbenzidine substrate solution (Thermo Scientific, Waltham, MA, USA), and the absorbance at $650 \mathrm{~nm}$ was measured using a Multiskan Ascent instrument (Labsystems, Helsinki, Finland).

\section{EDC crosslinking of cotinine to magnetic beads}

EDC (1-ethyl-3-(3-dimethylaminopropyl) carbodiimide; Thermo Scientific) was used to conjugate the carboxyl groups of trans- $4^{\prime}-$ cotininecarboxylic acid to the amine group on magnetic beads 
(Dynabeads M-270 Amine, Invitrogen) according to the manufacturer's instructions. Briefly, $8 \times 10^{7}$ beads, $52.8 \mu \mathrm{g}(240 \mathrm{nmol})$ trans$4^{\prime}$-cotininecarboxylic acid and $137.6 \mu \mathrm{g}(720 \mathrm{nmol})$ EDC were mixed in $800 \mu \mathrm{l}$ dimethylformamide and incubated for $2 \mathrm{~h}$ at room temperature with slow tilt rotation. Remaining reagents were removed by repeated washes with dimethylformamide.

\section{Immunoprecipitation of $\mathrm{C} 5$ in human serum}

Human serum $(200 \mu \mathrm{l})$ was preincubated overnight with $35 \mu \mathrm{l}$ protein A agarose beads (RepliGen) to remove Ig. After centrifugation of preincubated serum at 2500 r.p.m. at $4{ }^{\circ} \mathrm{C}$, the supernatant was incubated with $1 \mu \mathrm{g}$ anti-C5 $\times$ anti-cotinine bispecific tandem scFv-Fc fusion protein for $4 \mathrm{~h}$ at $4{ }^{\circ} \mathrm{C}$ with constant rotation. Control samples, without either serum or bispecific tandem $\mathrm{scFv}$-Fc fusion protein, were incubated in parallel. Immune complexes were then precipitated by addition of cotinine-crosslinked magnetic beads $\left(8 \times 10^{7}\right.$ beads $)$ or protein A agarose beads $(20 \mu \mathrm{l})$ and incubated for $1 \mathrm{~h}$ at $4{ }^{\circ} \mathrm{C}$ with constant rotation. Beads were washed once with PBS and three times with PBS containing 1\% Triton X-100 (Sigma-Aldrich), resuspended in NuPAGE LDS Sample Buffer (Invitrogen) and boiled for $5 \mathrm{~min}$, and separated by electrophoresis for immunoblot analysis.

\section{Immunoblot analysis}

Human serum were diluted $1 / 20,1 / 40$ and $1 / 80$ with PBS and electrophoresed under nonreducing conditions in a SDS-PAGE (NuPAGE 4-12\% Bis-Tris, Invitrogen) gel and transferred to a nitrocellulose membrane (Whatman, Kent, UK) as reported previously. ${ }^{6}$ Human complement C5 (Merck Millipore) served as a positive control.

The blot was blocked with 5\% skim milk in PBS containing 0.02\% Tween-20 (Sigma-Aldrich) for $1 \mathrm{~h}$ at room temperature and then incubated with $2 \mu \mathrm{g} \mathrm{ml}^{-1}$ anti-C5 $\times$ anti-cotinine bispecific tandem $\mathrm{scFv}-\mathrm{Fc}$ fusion protein overnight at $4{ }^{\circ} \mathrm{C}$. The blot was then washed five times in PBS containing 0.02\% Tween-20 and incubated with either cotinine-HRP $\left(1 \mu \mathrm{g} \mathrm{ml}^{-1}\right)$ or HRP-conjugated goat anti-rabbit IgG (Fc-specific) antibody (100 $\mathrm{ng} \mathrm{ml}^{-1}$; Abcam, Cambridge, UK) for
$2 \mathrm{~h}$ at room temperature. Bands were visualized using an enhanced chemiluminescence system (Thermo Scientific) according to the manufacturer's instructions.

\section{RESULTS}

\section{Preparation and characterization of bispecific tandem scFv-Fc fusion protein}

A gene construct encoding the anti-C $5 \times$ anti-cotinine bispecific tandem scFv-Fc fusion protein was cloned into a eukaryotic expression vector for expression in mammalian cells. We used the leader sequence of the human Ig $\kappa$-chain so that the recombinant protein would be secreted by the transfected cells into the culture medium. Because the Fc domain provides a protein A-binding domain, relatively pure recombinant protein could be obtained by protein A affinity chromatography. After purification, the major protein of $80 \mathrm{kDa}$ was observed, in agreement with expectations (Figure $2 \mathrm{~b}$ ). To check whether the anti-C5 $\times$ anti-cotinine bispecific tandem scFv-Fc fusion protein binds to complement C5 and cotinine simultaneously, we performed an enzyme immunoassay using a microtiter plate coated with complement C5 (Figure 3). After addition of anti$\mathrm{C} 5 \times$ anti-cotinine bispecific tandem $\mathrm{scFv}-\mathrm{Fc}$ fusion protein, either cotinine-HRP or HRP-conjugated goat anti-rabbit IgG (Fc-specific) antibody was added to individual wells. In the wells incubated with cotinine-HRP, the anti-C5 $\times$ anti-cotinine bispecific tandem $\mathrm{scFv}-\mathrm{Fc}$ fusion protein showed a dose-dependent reactivity in the range of $4-4000 \mu \mathrm{g} \mathrm{ml}^{-1}$. No significant nonspecific reactivity was observed in control wells blocked with skim milk.

\section{Bispecific tandem scFv-Fc fusion protein reduces background staining in serum immunoblot}

To compare the level of background generated by cotinineHRP with that generated by a traditional secondary antibody,

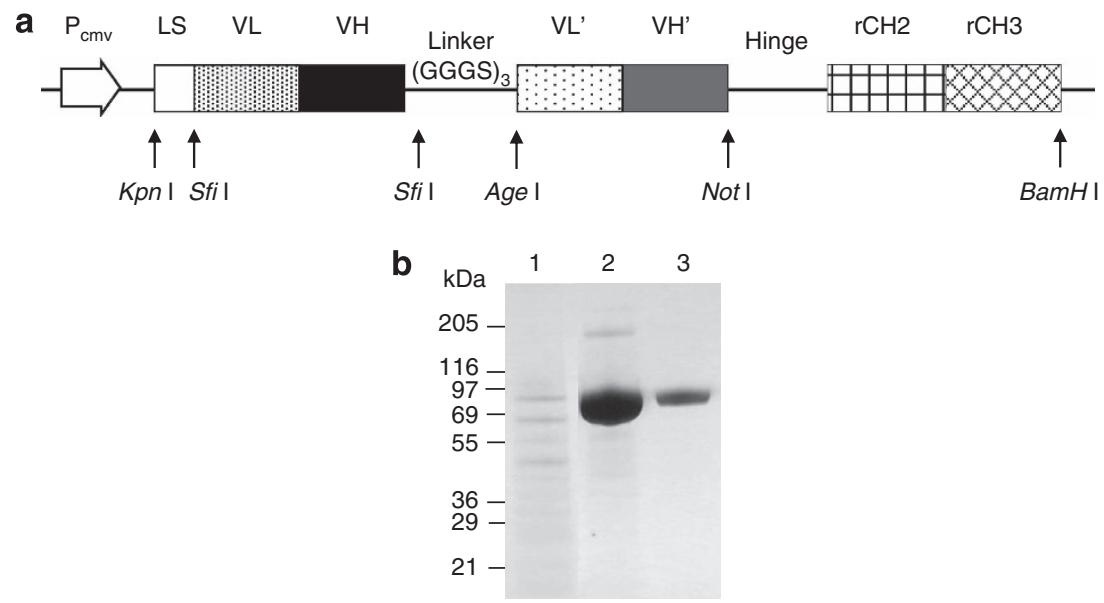

Figure 2 Gene construct and purification of bispecific tandem single-chain variable fragment (scFv)-Fc fusion protein. (a) Arrangement of genes in the pCEP4 vector. $P_{\mathrm{cmv}}$ denotes the cytomegalovirus promoter. LS denotes the leader sequence of the human immunoglobulin (Ig) $\kappa$-chain. VL and VH denote the variable light and heavy chains of Ig, respectively. A linker consisting of (Gly-Gly-Gly-Ser) 3 was inserted between the two scFvs. Hinge denotes the hinge sequence of human $\operatorname{lgG}_{1}$. Two constant domains of rabbit IgG, $\mathrm{C}_{\mathrm{H} 2}$ and $\mathrm{C}_{\mathrm{H} 3}$, were employed. (b) Anti-C5 $\times$ anti-cotinine bispecific tandem scFv-Fc fusion protein was purified by protein A affinity chromatography and subjected to sodium dodecyl sulfate-polyacrylamide gel electrophoresis (SDS-PAGE) analysis. Lane 1 was loaded with the flow-through fraction. Lanes 2 and 3 were loaded with purified protein at two different concentrations. 

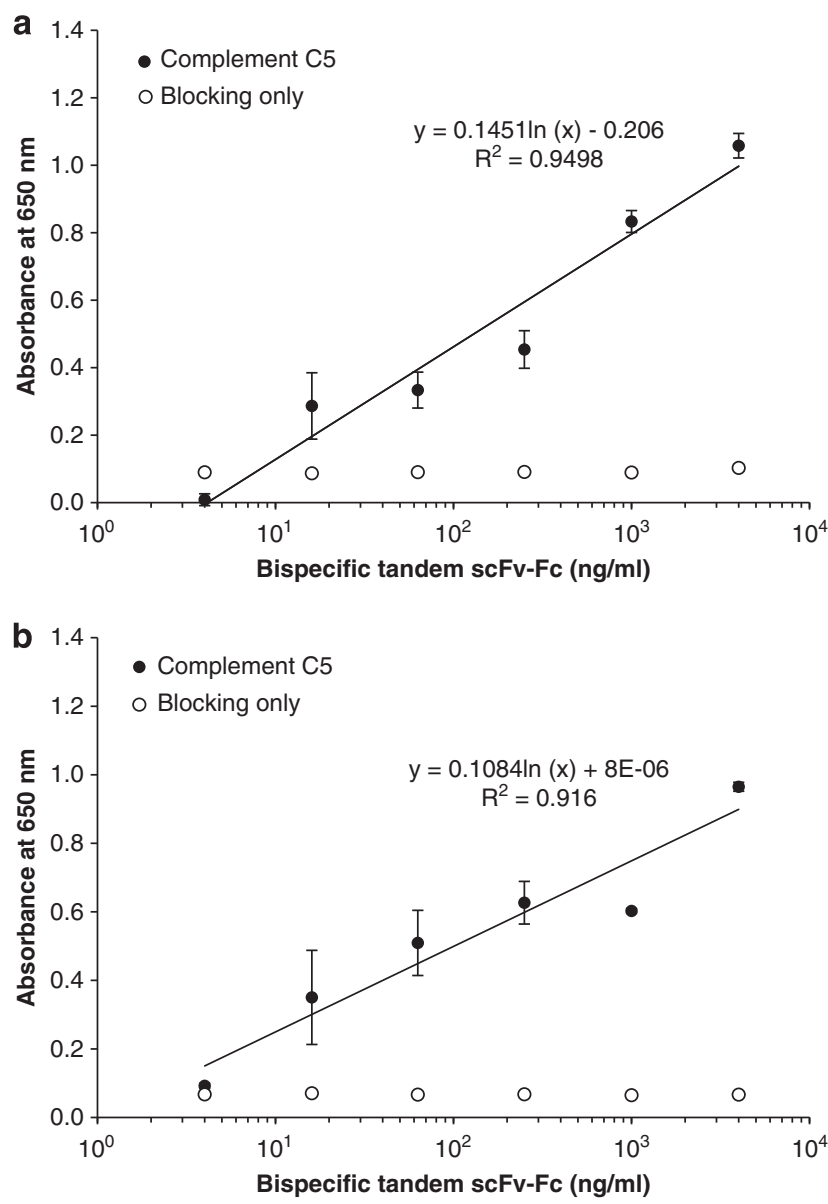

Figure 3 Enzyme immunoassay using bispecific tandem singlechain variable fragment (scFv)-Fc fusion protein. (a) Anti-C5 $\times$ anticotinine bispecific tandem scFv-Fc fusion protein was added to wells of a microtiter plate coated with complement $\mathrm{C} 5$, and probed with cotinine-horseradish peroxidase (HRP). Background signal was measured in control wells that were not coated with complement C5. (b) Anti-C5 $\times$ anti-cotinine bispecific tandem scFv-Fc fusion protein bound to complement C5 was probed with HRP-conjugated goat anti-rabbit IgG (Fc-specific) antibody. Background signal was measured in control wells that were not coated with complement C5. The results are expressed as the mean \pm s.d. of triplicate measurements.

human serum was diluted $1 / 20,1 / 40$ and $1 / 80$ with PBS and subjected to immunoblot analysis with the bispecific anti-C5 $\times$ anti-cotinine tandem $\mathrm{scFv}-\mathrm{Fc}$ fusion protein and cotinine-HRP or HRP-conjugated goat anti-rabbit IgG (Fc-specific) antibody.

Human complement C5, corresponding to a molecular mass of $190 \mathrm{kDa}$, was clearly visualized using the anti-C $5 \times$ anti-cotinine bispecific tandem $\mathrm{scFv}-\mathrm{Fc}$ fusion protein and cotinine-HRP without significant background (Figure $4 \mathrm{a}$, left). A blot incubated with cotinine-HRP only showed no signal, confirming the absence of detectable protein in human serum that interacts with cotinine or HRP (Figure $4 \mathrm{~b}$, left). In contrast, a high level of background was seen on the blot probed with HRP-conjugated goat anti-rabbit IgG
(Fc-specific) antibody (Figures $4 \mathrm{a}$ and $\mathrm{b}$, right). This comparative experiment clearly demonstrated that the significant background generated by HRP-conjugated goat anti-rabbit IgG (Fc-specific) antibody can be drastically reduced by the use of cotinine-HRP.

\section{Cotinine-crosslinked magnetic beads precipitate immune complexes with reduced contaminants}

To compare the amount of contaminants coprecipitated by cotinine-crosslinked magnetic beads and by protein A beads, the mixture of human serum and anti-C $5 \times$ anti-cotinine bispecific tandem $\mathrm{scFv}$-Fc fusion protein was incubated with each solid support in parallel. After the magnetic beads and protein A beads were washed, the proteins bound to both supports were eluted by addition of SDS-PAGE sample buffer and boiling. The eluate was subjected to SDS-PAGE and immunoblot analysis with anti-C $5 \times$ anti-cotinine bispecific tandem $\mathrm{scFv}-\mathrm{Fc}$ fusion protein as the primary antibody and HRP-conjugated goat anti-rabbit IgG (Fc-specific) antibody.

On the blot containing samples immunoprecipitated with protein $\mathrm{A}$ beads and probed with the anti-C $5 \times$ anti-cotinine fusion protein $\left(2 \mu \mathrm{g} \mathrm{ml}^{-1}\right)$ and HRP-conjugated goat antirabbit IgG (Fc-specific) antibody $\left(100 \mathrm{ng} \mathrm{ml}^{-1}\right)$, significant background could be observed (Figure 5a, left panel), which is thought to have originated from binding of HRP-conjugated secondary antibody to serum Ig (Figure $5 \mathrm{~b}$, left panel). When the cotinine-crosslinked magnetic beads were used for immunoprecipitation, the background was dramatically reduced, and only the bands corresponding to the anti-C $5 \times$ anticotinine fusion protein were visualized (Figure $5 \mathrm{a}$, middle panel). Replacement of the HRP-conjugated secondary antibody with cotinine-HRP $\left(1 \mu \mathrm{g} \mathrm{ml}^{-1}\right)$ as the secondary probe successfully eliminated the anti-C5 $\times$ anti-cotinine fusion protein band (Figures $5 \mathrm{a}$ and $\mathrm{b}$, right panel) because the reactivity of the bispecific fusion protein to cotinine was eliminated by boiling during the sample preparation step.

\section{DISCUSSION}

Immunoanalysis of antigens in biological materials is generally used to detect and analyze the dynamics of constituent proteins. The challenge in immunoblot analysis of biological samples lies in the heterogeneity of proteins in these samples, not only in terms of diversity but also in wide dynamic range. Up to 10000 proteins are commonly present in serum. ${ }^{7}$ The dynamic range of serum protein concentration extends from that of human serum albumin $\left(>45 \mathrm{mg} \mathrm{ml}^{-1}\right)$ and the Igs $\left(\sim 20 \mathrm{mg} \mathrm{ml}^{-1}\right)$ to some cytokines $\left(1-10 \mathrm{pg} \mathrm{ml}^{-1}\right) .^{8}$ In immunoblot analysis, membranes are sequentially exposed to primary antibody against a specific protein and enzyme-labeled secondary antibody against the constant region of the primary antibody. Because the constant regions of human and other species, such as mouse and rabbit, are highly homologous, and Ig is present at very high concentrations in serum and plasma, high background arising from the interaction of the secondary antibody and human $\mathrm{Ig}$ is frequently encountered in immunoblot analysis of these samples. We previously 


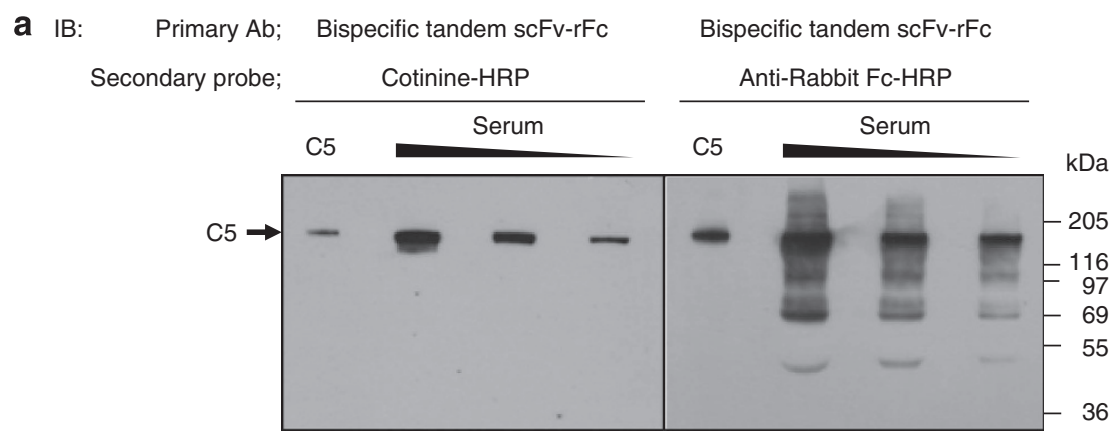

b IB: Secondary probe;
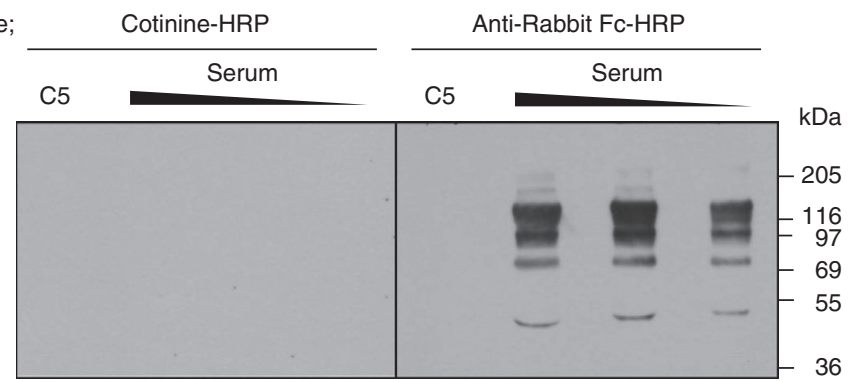

Figure 4 Comparison of the new and conventional immunoblot (IB) methods for probing complement C5 in human serum. Arrows indicate human complement C5 (190 kDa). (a) Immunoblot was probed with the bispecific tandem single-chain variable fragment (scFv)Fc fusion protein recognizing both C5 and cotinine and then with cotinine-horseradish peroxidase (HRP) (left) or HRP-conjugated goat anti-rabbit IgG (Fc-specific) antibody (right). (b) The same set of blots was incubated only with cotinine-HRP (left) or HRP-conjugated goat anti-rabbit IgG (Fc-specific) antibody (right).

developed an antibody against complement $\mathrm{C} 5$ and, during the process of characterizing its reactivity to complement $\mathrm{C} 5$ of various animal species by immunoblot analysis, encountered the problem of high background.

To overcome this issue, we employed the concept of a bispecific antibody reactive to antigen and a hapten. Historically, bispecific antibodies have been developed in diverse formats. ${ }^{9}$ Earlier versions of bispecific antibodies were generated by hybrid hybridoma technology, which enabled coexpression of two different IgG specificities. ${ }^{10}$ The hybrid antibodies, termed TriomAbs, were generated by the pairing of rat $\operatorname{IgG}_{2 \mathrm{a}}$ and mouse $\mathrm{IgG}_{2 \mathrm{~b}}$ to decrease the level of heterogeneity. ${ }^{11}$ Heterodimerization of heavy chains that contain a modification of the $\mathrm{C}_{\mathrm{H} 3}$ domain by knobs-intoholes technology yields IgG-like bispecific antibodies. ${ }^{12}$ The diabody has two scFvs with a short linker that allows interchain domain pairing. ${ }^{13}$ Other bispecific antibody formats, termed BiTE (bispecific T-cell engager) and DART (dual-affinity re-targeting), are based on the covalent linkage of two single-chain antibodies by a peptide linker ${ }^{14}$ and a disulfide bridge, ${ }^{15}$ respectively. For in vivo use, various formats of scFv fusion proteins, such as scFv-IgG, ${ }^{16} \mathrm{scFv}-\mathrm{Fc}_{-} \mathrm{scFv}^{17}$ and tandem $\mathrm{scFv}-\mathrm{Fc},{ }^{18}$ were developed because the $\mathrm{Fc}$ domain provided both prolonged pharmacokinetic half-life and Fcmediated effector function.

In this study, we tested several formats of bispecific antibody (data not shown) and finally adopted the bispecific tandem scFvs joined by a linker and fused to the Fc domain. The main advantage of this format is that four variable domains and one Fc domain are aligned on a single polypeptide chain encoded by just one gene. This format allows us to purify the fusion protein without concern for contaminating heterogeneous antibody structures that are common in other formats of bispecific antibody. ${ }^{19}$ The coexpression of $\mathrm{Fc}$ domain at the carboxy terminal of the bispecific tandem $\mathrm{scFv}$ as a fusion protein not only increased the expression yield (data not shown), but also had the advantage of enabling purification by affinity chromatography using protein A beads. It is clear that linkers and hinge residues provided sufficient flexibility for the bispecific tandem scFv-Fc fusion protein to bind to complement $\mathrm{C} 5$ and cotinine simultaneously, while preserving reactivity to protein A and secondary antibody (Figure 3).

Cotinine is a small chemical with a molecular mass of 176.22 and is the major metabolite of nicotine. Several characteristics make it an ideal hapten. First, it is not present in human and animal tissues, except in the case of tobacco exposure. ${ }^{4}$ It is well known that humans and other animals express no cotinine-binding molecules, and cotinine is physiologically inert. ${ }^{20}$ Carboxy cotinine is available commercially at low cost, and the carboxy group can be employed for chemical crosslinking. The anticotinine antibody we generated was shown to be specific for cotinine and did not crossreact with chemicals with similar structures such as nicotine, anabasine, caffeine and cholesterol. ${ }^{4}$ We previously showed that this anti-cotinine antibody successfully combined with cotinine-conjugated 


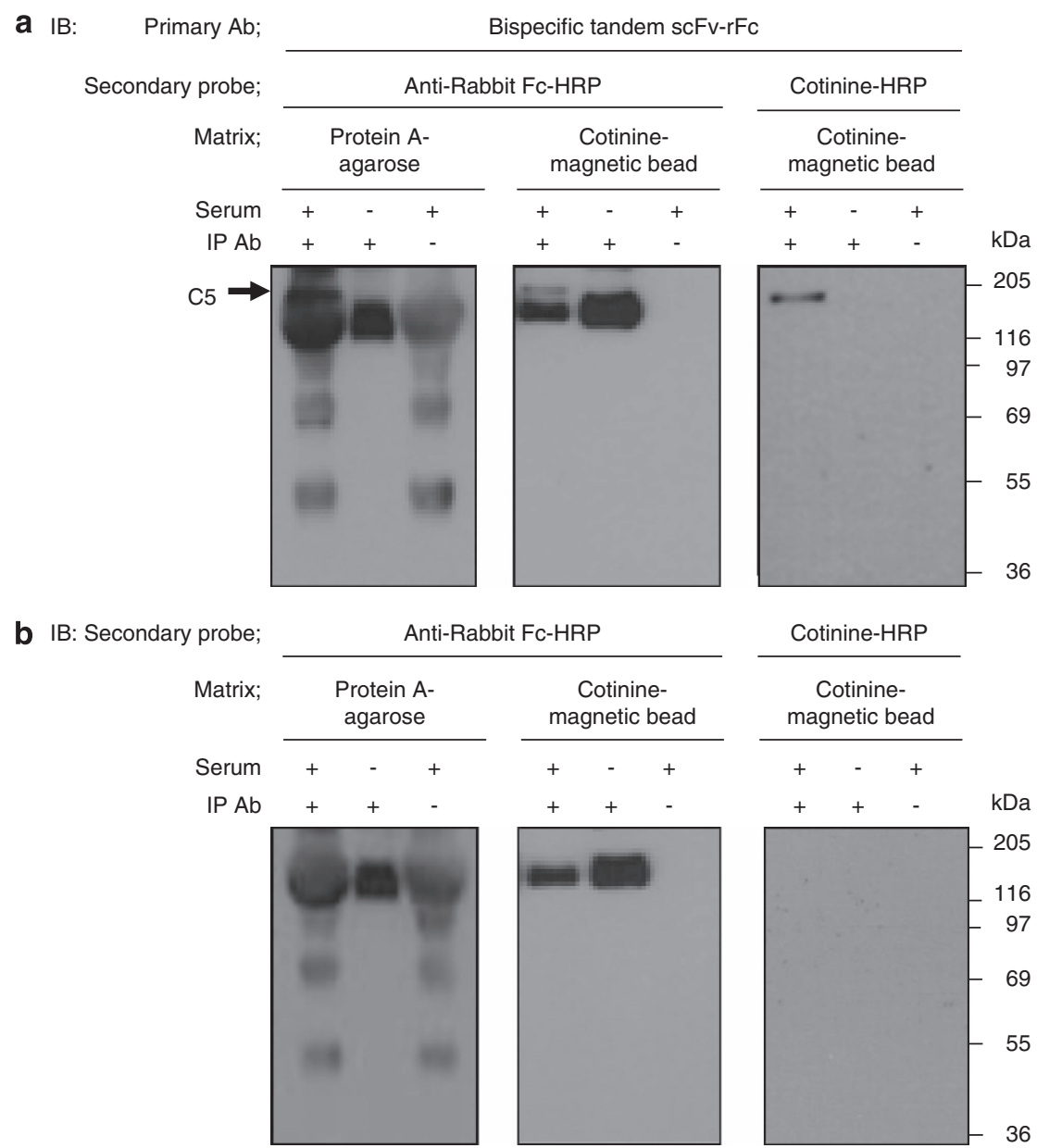

Figure 5 Decreased background staining with new immunoblot (IB) method coupled with application of magnetic beads in immunoprecipitation (IP). Immune complexes were precipitated either with protein A agarose beads (left panels) or cotinine-crosslinked magnetic beads (middle and right panels). (a) Blots were incubated with anti-C $5 \times$ anti-cotinine bispecific tandem scFv-Fc fusion protein and then probed with either horseradish peroxidase (HRP)-conjugated goat anti-rabbit IgG (Fc-specific) antibody (left and middle panels) or cotinine-HRP (right panel). (b) The control blots were incubated with secondary probes only, HRP-conjugated goat anti-rabbit IgG (Fcspecific) antibody (left and middle panels) or cotinine-HRP (right panel).

nucleic acid aptamers for use in immunoblotting and immunoprecipitation. ${ }^{20}$

In this study we showed that the anti-C5 $\times$ anti-cotinine bispecific tandem scFv-Fc fusion protein simultaneously recognizes complement $\mathrm{C} 5$ and cotinine, and that the $\mathrm{Fc}$ domain retains its reactivity to secondary antibody and protein A. We think that the versatility of this bispecific tandem scFvFc fusion protein will allow it to be used in a wide array of biological experiments.

\section{CONFLICT OF INTEREST}

The authors declare no conflict of interest.

\section{ACKNOWLEDGEMENTS}

This research was supported by Basic Science Research Program through the National Research Foundation of Korea (NRF) funded by the Ministry of Education (2009-0093820). This work was supported by the National Research Foundation of Korea (NRF) grant funded by the Korea government (MSIP) (2011-0030119).
1 Diamandis EP, Christopoulos TK. The biotin-(strept)avidin system: principles and applications in biotechnology. Clin Chem 1991; 37: 625-636.

2 Hall JM, Adams S, Williams S, Rehse MA, Layton TJ, Molesh DA. Purification of fetal cells from maternal blood using an avidin-biotin immunoaffinity column. Ann NY Acad Sci 1994; 731: 115-127.

3 Zempleni J, Wijeratne SS, Hassan YI. Biotin. Biofactors 2009; 35: 36-46.

4 Park S, Lee DH, Park JG, Lee YT, Chung J. A sensitive enzyme immunoassay for measuring cotinine in passive smokers. Clin Chim Acta 2010; 411: 1238-1242.

5 Boussif O, Lezoualc'h F, Zanta MA, Mergny MD, Scherman D, Demeneix B et al. A versatile vector for gene and oligonucleotide transfer into cells in culture and in vivo: polyethylenimine. Proc Natl Acad Sci USA 1995; 92: 7297-7301.

6 Burnette WN. "Western blotting": electrophoretic transfer of proteins from sodium dodecyl sulfate-polyacrylamide gels to unmodified nitrocellulose and radiographic detection with antibody and radioiodinated protein $\mathrm{A}$. Anal Biochem 1981; 112: 195-203.

7 Adkins JN, Varnum SM, Auberry KJ, Moore RJ, Angell NH, Smith RD et al. Toward a human blood serum proteome: analysis by multidimensional separation coupled with mass spectrometry. Mol Cell Proteomics 2002; 1: 947-955.

8 Jacobs JM, Adkins JN, Qian WJ, Liu T, Shen Y, Camp DG 2nd et al. Utilizing human blood plasma for proteomic biomarker discovery. J Proteome Res 2005; 4: 1073-1085. 
9 Muller D, Kontermann RE. Bispecific antibodies for cancer immunotherapy: current perspectives. Biodrugs 2010; 24: 89-98.

10 Milstein C, Cuello AC. Hybrid hybridomas and their use in immunohistochemistry. Nature 1983; 305: 537-540.

11 Lindhofer H, Mocikat R, Steipe B, Thierfelder S. Preferential speciesrestricted heavy light-chain pairing in rat mouse quadromas - implications for a single-step purification of bispecific antibodies. J Immunol 1995; 155: 219-225.

12 Ridgway JBB, Presta LG, Carter P. 'Knobs-into-holes' engineering of antibody $\mathrm{C}(\mathrm{H}) 3$ domains for heavy chain heterodimerization. Protein Eng 1996; 9: 617-621.

13 Holliger P, Prospero T, Winter G. "Diabodies": small bivalent and bispecific antibody fragments. Proc Natl Acad Sci USA 1993; 90: 6444-6448.

14 Wolf E, Hofmeister R, Kufer P, Schlereth B, Baeuerle PA. BiTEs: bispecific antibody constructs with unique anti-tumor activity. Drug Discov Today 2005; 10: 1237-1244.

15 Johnson S, Burke S, Huang L, Gorlatov S, Li H, Wang W et al. Effector cell recruitment with novel $\mathrm{Fv}$-based dual-affinity re-targeting protein leads to potent tumor cytolysis and in vivo B-cell depletion. J Mol Biol 2010; 399: 436-449.
16 Marvin JS, Zhu Z. Recombinant approaches to IgG-like bispecific antibodies. Acta Pharmacol Sin 2005; 26: 649-658.

17 Jendreyko N, Popkov M, Beerli RR, Chung J, McGavern DB, Rader C et al. Intradiabodies, bispecific, tetravalent antibodies for the simultaneous functional knockout of two cell surface receptors. J Biol Chem 2003; 278: 47812-47819.

18 Connelly RJ, Hayden MS, Scholler JK, Tsu TT, Dupont B, Ledbetter JA et al. Mitogenic properties of a bispecific single-chain Fv-lg fusion generated from CD2-specific mAb to distinct epitopes. Int Immunol 1998; 10: 1863-1872.

19 Chan AC, Carter PJ. Therapeutic antibodies for autoimmunity and inflammation. Nat Rev Immunol 2010; 10: 301-316.

20 Park S, Hwang D, Chung J. Cotinine-conjugated aptamer/anti-cotinine antibody complexes as a novel affinity unit for use in biological assays. Exp Mol Med 2012; 44: 554-561.

$(1) \Theta$ This work is licensed under a Creative Commons Attribution-NonCommercial-NoDerivs 3.0 Unported License. To view a copy of this license, visit http:// creativecommons.org/licenses/by-nc-nd/3.0/ 